\title{
INTRODUCTION TO QUANTUM COMPUTATION AND INFORMATION
}


This page is intentionally left blank 


\title{
INTRODUCTION TO QUANTUM COMPUTATION AND INFORMATION
}

\author{
Editors \\ Hoi-Kwong Lo \\ MagiQ Technologies, Inc., New York \\ Sandu Popescu \\ University of Bristol \& \\ BRIMS, Hewlett-Packard Laboratories, Bristol \\ Tim Spiller \\ Hewlett-Packard Laboratories, Bristol

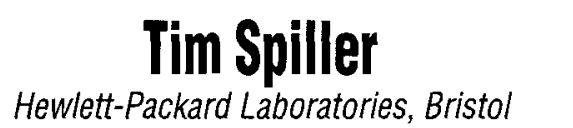


Published by

World Scientific Publishing Co. Pte. Ltd.

P O Box 128, Farrer Road, Singapore 912805

USA office: Suite 1B, 1060 Main Street, River Edge, NJ 07661

UK office: 57 Shelton Street, Covent Garden, London WC2H 9HE

\author{
Library of Congress Cataloging-in-Publication Data \\ Lo, Hoi-Kwong. \\ Introduction to Quantum computation and information / Hoi-Kwong Lo \\ \& Tim Spiller, Sandu Popescu. \\ p. $\mathrm{cm}$. \\ Includes bibliographical references. \\ ISBN 981023399X ISBN 981024410X (pbk) \\ 1. Quantum computers. I. Spiller, Tim. II. Popescu, Sandu. \\ III. Title. \\ QA76.889.L6 1998 \\ $004.1--d c 21$ \\ 98-31095 \\ CIP

\section{British Library Cataloguing-in-Publication Data} \\ A catalogue record for this book is available from the British Library.
}

First published 1998

Reprinted 1999, 2000

Copyright (C) 1998 by World Scientific Publishing Co. Pte. Ltd.

All rights reserved. This book, or parts thereof, may not be reproduced in any form or by any means, electronic or mechanical, including photocopying, recording or any information storage and retrieval system now known or to be invented, without written permission from the Publisher.

For photocopying of material in this volume, please pay a copying fee through the Copyright Clearance Center, Inc., 222 Rosewood Drive, Danvers, MA 01923, USA. In this case permission to photocopy is not required from the publisher.

Printed in Singapore by Uto-Print 


\title{
FOREWORD
}

\author{
MICHAEL BERRY \\ H. H. Wills Physics Laboratory, University of Bristol \\ Tyndall Avenue, Bristol BS8 1 TL, United Kingdom
}

In the nineteenth century, life was transformed by the conscious application of classical mechanics, in the form of Newton's equations (and, later, thermodynamics) to the engines of the industrial revolution. In this century, a similar transformation has been wrought by electromagnetism, in generating and distributing electric power and communicating words and pictures across the world at the speed of light, in what should be seen as the conscious application of Maxwell's equations. It is easy to predict that in the twenty-first century it will be quantum mechanics that influences all our lives.

There is a sense in which quantum mechanics is already having profound effects. Leon Lederman claims that a large part of the gross national product of the industrialized countries stems from quantum mechanics. I suppose he is referring to transistors - the 'fundamental particles' of modern electronics - that depend on properties of semiconducting materials designed by applying quantum mechanics to electrons in solids, and to lasers, where the Bose-Einstein statistics of identical particles generates coherent avalanches of photons to read the bar-codes in our supermarkets and guide delicate surgery in our eyes.

The most dramatic influences are, however, likely to come from the deliberate manipulation of entangled states. These arise naturally when Thomas Young's superposition principle, familiar throughout wave physics since 1800, is generalized to states of more than one particle. Entanglement lies at the heart of the microscopic world as described by quantum mechanics, and makes it weirdly different from the world of our immediate experience. Schrödinger invented the idea of entanglement more than sixty years ago (although a version of it had appeared before, in the quantum states of identical particles); but only now, in a remarkable flowering of fin de siècle quantum mechanics, are its full implications being thoroughly and energetically explored. There is much to understand; even a convincing measure of entanglement is lacking.

In an entangled state of several particles, measurements on one particle can affect all the others, even if they are too far apart for a causal influence to propagate between them. These nonlocal (but not relativity-violating) actions are being incorporated into proposals for technologies that were hardly imagined twenty years ago. Most of the attention is being focused on quantum 
computing, quantum cryptography, and teleportation.

In quantum computing, information is manipulated not discretely, in the classical way, as a series of zeros and ones (bits), but as continuous superpositions (qubits) where the number of possibilities is vastly greater. In effect, many computations are performed simultaneously, and calculations that would be intractable classically (for example, factoring large integers) become feasible quantally. In this way, the theory of computation-indeed information science itself-is becoming a branch of physics rather than mathematics.

Easy factorization would destroy one of the commonly-used methods of encryption. However, the same entanglement employed in quantum computing makes possible the development of unbreakable shared codes, incorporating the intrinsic randomness of quantum mechanics. To my mind, this particular emphasis in the application of fundamental physics is depressing, because I regard the obsession with secrets in public life (as opposed to a commendable discretion about private matters) as one of the less attractive preoccupations of our fellow human beings.

Teleportation is the dissolution of an object in one place in a way that enables it to be perfectly reconstituted elsewhere. On the finest scale, the most complete specification of an object is its quantum state, but complete knowledge of that cannot be had: measurement of one property of the state irrevocably destroys information about complementary properties. However, suitable measurements can entangle the teleportee (whose state is unknown) with one of a previously entangled pair of systems, and thereby transfer the state to the other member of the pair, however far away that is, where another measurement (requiring information sent conventionally) can reconstruct it.

Each of these projects is visionary - technological fantasy, some say-but the principles have been demonstrated experimentally. The big obstacle to further developments is 'decoherence', in which uncontrolled effects of the environment scramble the delicate phase correlations that embody quantum entanglement. Decoherence has plagued wave physics from its beginnings; it is what made Thomas Young's superpositions, in his double-slit experiments with light, so hard to create and maintain (his experiments were carried out with candles!). States of many particles are even more fragile. Recent work suggests that the effects of decoherence might be reduced or eliminated by cleverly correcting errors as they arise.

This book is a record of these modern developments, a self-contained pedagogical account-perhaps the first-written by the world's leading experts. Most of the chapters were 'battle-tested' in a series of lectures at HewlettPackard's Basic Research Institute in the Mathematical Sciences (BRIMS) in Bristol, United Kingdom. That the lectures were sponsored by Hewlett- 
Packard indicates the intense industrial interest in a branch of theoretical, and, increasingly, experimental, physics that optimists (including me) believe is also a nascent technology. 
This page is intentionally left blank 


\title{
PREFACE
}

\author{
HOI-KWONG LO \\ MagiQ Technologies, Inc. \\ 275 Seventh Avenue, 26th Floor, \\ New York, NY 10001-6708, USA \\ TIMOTHY P. SPILLER \\ Hewlett-Packard Laboratories, Bristol \\ Filton Road, Stoke Gifford \\ Bristol BS34 8QZ, United Kingdom \\ SANDU POPESCU \\ University of Bristal, Tyndall Avenue \\ Bristol BS8 1TL, United Kingdom \\ and \\ BRIMS, Hewlett-Packard Laboratories, Bristol \\ Filton Road, Stoke Gifford, \\ Bristol BS34 8QZ, United Kingdom
}

\begin{abstract}
About This Book:
This book is an introduction to quantum information and what can be done with it: computation, teleportation and cryptography. Following the pioneering work by Richard Feynman, David Deutsch and others in the 1980's, there has been an explosive growth in the research activities in the whole area. It is now known that information processing based on the principles of quantum mechanics can be profoundly different and, in some cases, much more powerful than that based on classical mechanics. Apart from technological interests, quantum computation and related subjects also provide a concrete arena for investigating fundamental issues in quantum mechanics.

Numerous exciting results and developments have emerged, experimental as well as theoretical, and many of them are discussed in this book. Topics covered include the non-locality of quantum mechanics, quantum teleportation, quantum computation, quantum cryptography, quantum error correction and fault-tolerant quantum computation, as well as some experimental aspects of quantum computation and quantum cryptography.

This book aims to be a self-contained overview. Only knowledge of basic quantum mechanics is assumed. Density matrices, irreversibility, entanglement and the like are discussed rather than simply taken as read. Concepts and
\end{abstract}


ideas from computational complexity theory, cryptology and error correcting codes are also introduced as needed. While important basic results are presented in detail, some more involved technical details are excluded. (Extensive reference lists are given with each chapter for readers who wish to go further.) The book should be well-suited for a wide audience, ranging from beginning graduate students to advanced researchers. Clearly the whole field of quantum information is still developing-no doubt as you are reading this, researchers somewhere in the world will be endeavouring to push the boundaries beyond those reported here. Nevertheless, the contents of this book should continue to provide a good primer for the further exciting breakthroughs that will surely follow in years to come.

Although the focus of industrial research laboratories is clearly on the technology and products of the near future, it also pays to invest some effort towards potential new long term technology, through basic and "blue skies" research. In 1994 Hewlett-Packard set up BRIMS, the Basic Research Institute in the Mathematical Sciences, ${ }^{a}$ which is part of Hewlett-Packard Laboratories Bristol (UK), (the European arm of the Company's corporate research structure). Members of BRIMS undertake basic research in various areas of mathematics and theoretical physics within this industrial setting. In addition, fundamental research in mathematics and physics is also carried out at Bristol in the Mathematics, Cryptography and Security Group, ${ }^{b}$ another part of the main HP Laboratories, in conjunction with their applied and consultative activities. The future potential for a whole new quantum information technology is clearly very exciting, (a point also highlighted by Michael Berry in his Foreword). Quantum information and computing are thus two of the basic research themes currently being pursued in BRIMS and the Mathematics, Cryptography and Security Group. This book is based on a series of lectures organized by the three editors under the auspices of BRIMS, from November 1996 to April 1997. In addition to speakers writing chapters based on their lectures, other contributions have been included. This widens the scope of the book and thus presents a good balanced overview of quantum information processing in general.

\section{About The Editors:}

Hoi-Kwong Lo is Chief Scientist and Senior Vice President, Research and Development of MagiQ Technologies, Inc., ${ }^{c}$ New York, a company founded in 1999 that focuses on the commercialization of quantum information technology in

\footnotetext{
${ }^{a}$ More information can be found at http://www-uk.hpl.hp.com/brims/

${ }^{b}$ More information can be found at http://www-uk.hpl.hp.com/mcs

${ }^{c}$ More information can be found at http://www.magiqtech.com
} 
addition to basic research on the subject. From 1996 to 1999, he worked at Hewlett-Packard Labs, Bristol (UK), first as a Research Consultant and subsequently as a Senior Member of Technical Staff. From 1994 to 1996, he was a Member of the School of Natural Sciences of the Institute for Advanced Study, Princeton (USA). He obtained his B.A. in Mathematics (Triple First Class Honour) from Trinity College, Cambridge University (UK) in 1989 and his M.S. and Ph.D. in Physics from the California Institute of Technology, Pasadena (USA) in 1991 and 1994 respectively.

Tim Spiller is currently a Technical Consultant in the Mathematics, Cryptography and Security Group, Hewlett-Packard Laboratories, Bristol (UK) and Coordinator of QUIPROCONE, the European Network of Excellence for Quantum Information Processing and Communication. ${ }^{d}$ He was formerly a UK Science and Engineering Research Council Research Fellow and a Royal Society Research Fellow in the Physics Department at the University of Sussex (UK). He got his B.A. in Physics from the University of Oxford (UK) in 1980 and his Ph.D. from the University of Durham (UK) in 1984.

Sandu Popescu currently holds a joint appointment as Professor of Physics at the University of Bristol and member of Hewlett-Packard's Basic Research Institute in the Mathematical Sciences (BRIMS) at Hewlett-Packard Laboratories, Bristol (UK). From 1996 to 1999 he was Hewlett-Packard Senior Research Fellow and University Reader in Quantum Mechanics at the Isaac Newton Institute, University of Cambridge (UK). He was formerly a Postdoctoral Fellow at the Free University of Bruxelles (Belgium) and at Boston University (USA). He got his B.A. in 1980 and M.Sc. in 1981, both at University of Bucharest, Romania and his Ph.D. in Physics from Tel-Aviv University (Israel) in 1991.

\section{Acknowledgments}

We thank all the authors for their considerable time and effort spent on writing up their manuscripts. We also thank Michael Berry for writing the Foreword to this book. We have benefitted from the generous support of both BRIMS and the Mathematics, Cryptography and Security Group at Hewlett-Packard Laboratories, Bristol, during the staging of the lectures and the preparation of the book. The help and service provided by World Scientific Publishing is also gratefully acknowledged.

\footnotetext{
${ }^{d}$ More information can be found at http://www.quiprocone.org
} 
This page is intentionally left blank 


\section{CONTENTS}

Foreword $\quad$ v

Preface $\quad$ ix

Basic Elements of Quantum Information Technology 1

Timothy P. Spiller

The Joy of Entanglement

Sandu Popescu and Daniel Rohrlich

Quantum Information and Its Properties

Richard Jozsa

Quantum Cryptology

Hoi-Kwong Lo

Experimental Quantum Cryptography

Hugo Zbinden

Quantum Computation: An Introduction

Adriano Barenco

Quantum Error Correction

Andrew M. Steane

Fault-Tolerant Quantum Computation

213

John Preskill

Quantum Computers, Error-Correction and Networking:

Quantum Optical Approaches

Thomas Pellizzari

Quantum Computation with Nuclear Magnetic Resonance

Isaac L. Chuang

Future Directions for Quantum Information Theory

Charles H. Bennett 